\title{
Elaboración y prueba de un electrodo para la cuantificación de cloruro de potasio y bromuro de potasio
}

\section{Development and testing of an electrode for determination of potassium chloride and potassium bromide}

José de Jesús Pérez-Saavedra'

Sonia Rincón-Arce ${ }^{2}$

Félix Fuentes-Armenta ${ }^{3}$

Fecha de recepción: 8 de enero del 2014

Fecha de aprobación: 27 de abril del 2014

Pérez-Saavedra, j; Rincón-Arce, S; Fuentes-Armenta,

F. Elaboración y prueba de un electrodo para la cuantificación de cloruro de potasio y bromuro de potasio. Tecnología en Marcha. Vol. 27, Nº 3, Julio-

Setiembre 20I4. Pág 80-87.

I Químico Farmacéutico, Biólogo. Doctorado en Ciencias Naturales para el Desarrollo. Facultad de Estudios Superiores CuautitlánUNAM. Teléfono: 52 (55)562320I3 México. Correo electrónico: quimicaa@unam.mx

2 Licenciada en Química. Facultad de Estudios Superiores Cuautitlán-UNAM. Teléfono: 52 (55)562320I 3 México. Correo electrónico: rincon@unam.mx

3 Licenciado en Ingeniería Química. Facultad de Estudios Superiores Cuautitlán-UNAM. Teléfono: 52 (55)562320I 3 México. Correo electrónico: pesaaje@yahoo.com.mx 


\section{Palabras clave}

Electrodos; plata; cloruros; bromuros; agar.

\section{Resumen}

Con la idea de mejorar el método potenciométrico para cuantificar cloruro de potasio y bromuro de potasio, se diseñó un electrodo combinado de plata como indicador y cobre/nitrato de cobre como referencia, donde los electrolitos del electrodo no interfieren en la cuantificación y al mismo tiempo se pueden disminuir los volúmenes por valorar (Arnaiz, 2005; Rincón y Pérez 2003), además de eliminar el puente de agar. A los resultados de los volúmenes de punto de equivalencia, con el montaje convencional y el electrodo elaborado, se les aplica la prueba estadística de $\mathrm{t}$ de Student con un 95\% de confianza (Harris, 1991), dando como resultado que no hay diferencia significativa, con ese nivel de confianza, entre ambos métodos.

\section{Key words}

Electrode; silver; chlorides; bromide; agar.

\section{Introducción}

De los métodos instrumentales que se utilizan en Química Analítica, la potenciometría a intensidad nula es uno de los métodos más amigables por varías y justificadas razones, por ejemplo, para medir $\mathrm{pH}$, basta con tener un potenciómetro y electrodo de vidrio combinado, calibrados, sumergir el electrodo en la solución problema y tomar la medida (Harris, 199I), esto se puede realizar tanto en un laboratorio convencional como en el campo (con un potenciómetro de baterías), esta instrumentación no necesita de instalaciones especiales, pero además es muy versátil puesto que con el potenciómetro se puede dar seguimiento de curvas de valoración ácido-base, redox, complejométricas y de halogenuros, determinación de parámetros termodinámicos como constantes de acidez, constantes de solubilidad, potenciales normales redox, cuantificación de metales con electrodos selectivos de iones e inclusive se pueden realizar estudios de cinética de reacciones químicas.

Dentro de estos métodos potenciométricos, se encuentra la cuantificación de Cloruros y Bromuros, utilizando un electrodo indicador de Plata, en combinación con un electrodo de referencia de Plata/

\begin{abstract}
To improve the potentiometric method for measuring potassium chloride and potassium bromide a combination electrode is designed, using as an indicator electrode Silver, and Copper/Copper nitrate as a reference electrode, where the electrolyte of the electrode do not interfere in the measurement and at the same time we can decrease to quantify volumes while eliminating salt bridge. In the results of the equivalence point volumes with conventional assembly and electrode developed, statistical Student's t-test with $95 \%$ significance level, is applied, resulting no significant difference with that level of reliance between methods.
\end{abstract}

Cloruro de plata, el cual para su funcionamiento óptimo, requiere estar saturado con una solución de Cloruro de potasio, este electrodo en la práctica convencional es separado de la solución por valorar mediante un puente de agar, esta separación debe hacerse ya que el electrodo de referencia mantiene el contacto (para cerrar el circuito eléctrico) con la solución a través de la migración de los iones de Cloruro y de Potasio a través de una frita porosa, esta movilidad iónica afecta la valoración por dos causas, la primera es que la migración de los iones Cloruro del electrodo de referencia, modifica la concentración de los iones Cloruro del sistema por valorar, la segunda es que si el electrodo referencia está en contacto directo con los iones Plata, la mayor concentración de iones Cloruro se encuentra en la proximidad de la frita porosa, donde al estar en contacto con los iones Plata, se forma un precipitado de Cloruro de plata que anula el contacto eléctrico, impidiendo la cuantificación.

Por tal motivo y para evitar la utilización de un puente de agar, el cual se tiene que elaborar cada ocasión que se realiza una valoración, lo que implica un problema que involucra tiempo, esfuerzo y recursos, se pensó en sustituir electrodo 


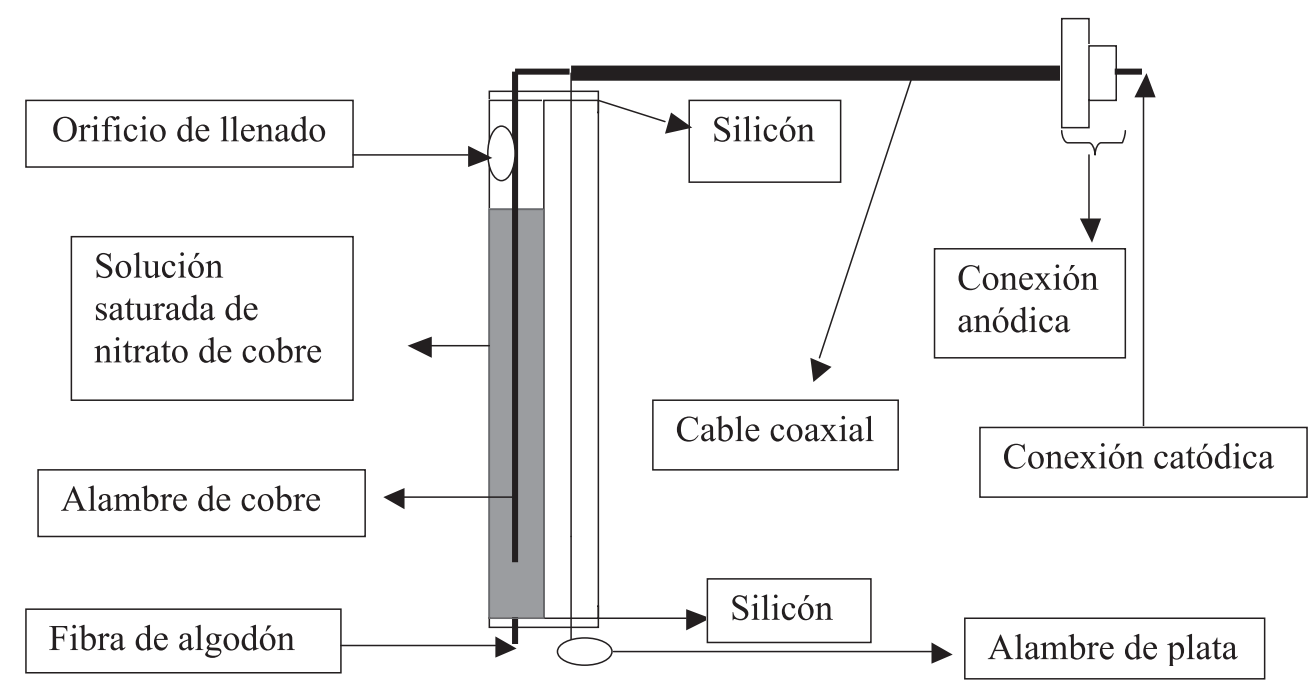

Figura I. Partes que conforman el electrodo elaborado

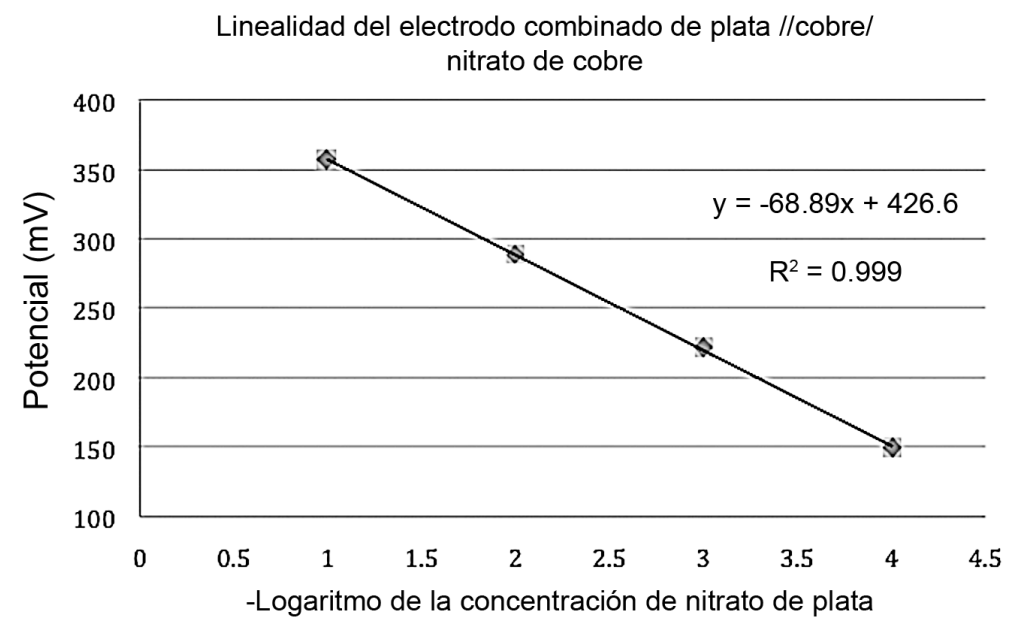

Figura 2. Curva de calibración de los potenciales promedio, medidos con el electrodo elaborado.

de referencia convencional de Plata/Cloruro de plata por un electrodo de Cobre/ Nitrato de cobre, de bajo costo y fácil elaboración, (Pérez y Rincón 2013), en donde la migración iónica se da con los iones Cobre y Nitrato, iones que no afectan la valoración de Bromuros ni de Cloruros.

\section{Objetivo}

Elaborar un electrodo combinado de plata como indicador y cobre/nitrato de cobre como referencia, para luego probar experimentalmente su funcionamiento en la microvaloración de bromuro de potasio y cloruro de potasio, evitando el uso del puente de agar.

\section{Metodología}

Elaboración del electrodo combinado

\section{a. Electrodo indicador de plata:}

Un alambre de plata calibre 30 de 2 centímetros es soldado al alambre interno de un cable coaxial (Pérez, Rincón y Tenorio, 2013) e introducido a un tubo de $4 \mathrm{~mm}$ de diámetro y $5 \mathrm{~cm}$ de altura, el cual es sellado en su parte inferior y superior con silicón (Charlot, Badoz y Tremillon, 1969). 
1. Bureta de vidrio de $5 \mathrm{~mL}$

2. Electrodo plata

3. Electrodo de calomel

4. Puente salino agar - agar

5. Electrodo combinado elaborado

6. Solución de cloruro de sodio

7. Potenciómetro Oakton

8. Potenciómetro Oakton

9. Agitador magnético

10. Barra magnética de $5 \times 2 \mathrm{~mm}$

11. Solución por valorar

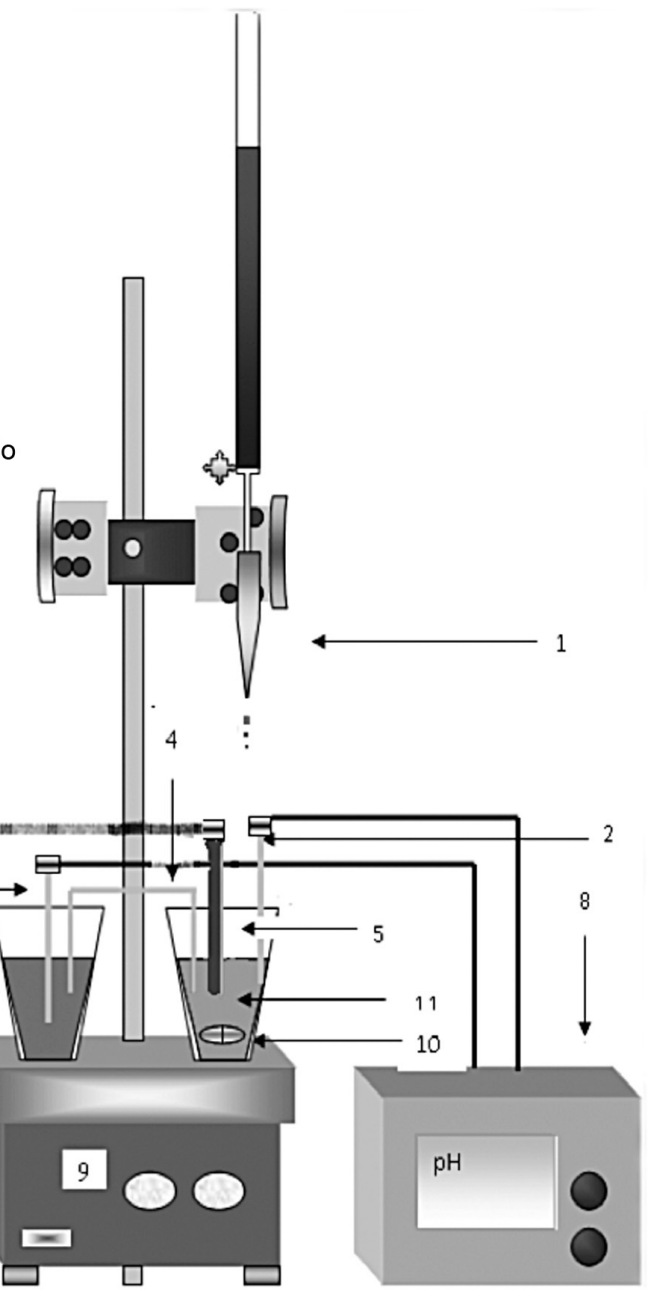

Figura 3. Montaje experimental de la valoración simultánea con ambos electrodos, el convencional y el elaborado.

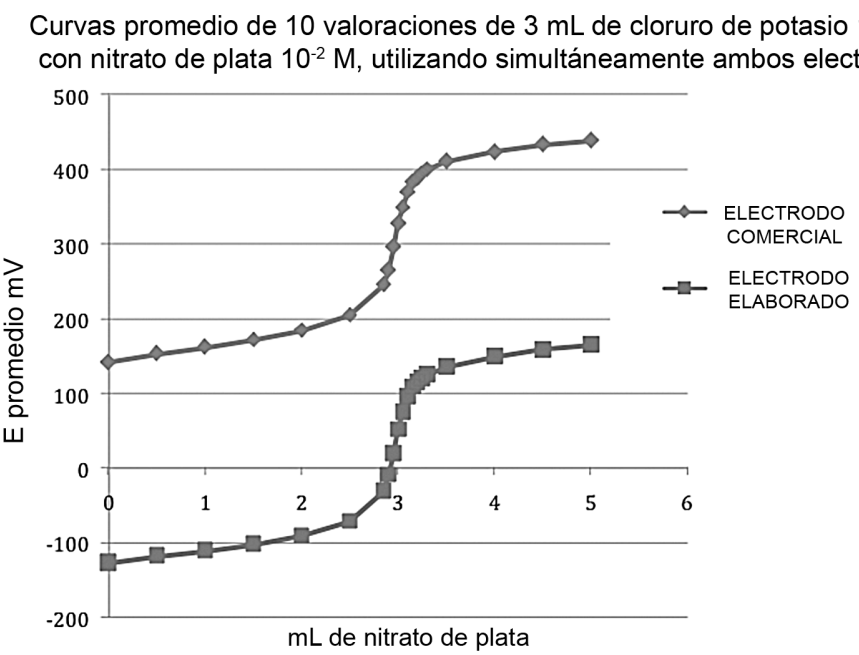

Figura 4. Curvas promedio de 10 valoraciones de cloruro de potasio 0,0 I M con nitrato de plata 0,0 IM, utilizando simultáneamente el electrodo comercial y el electrodo elaborado. 


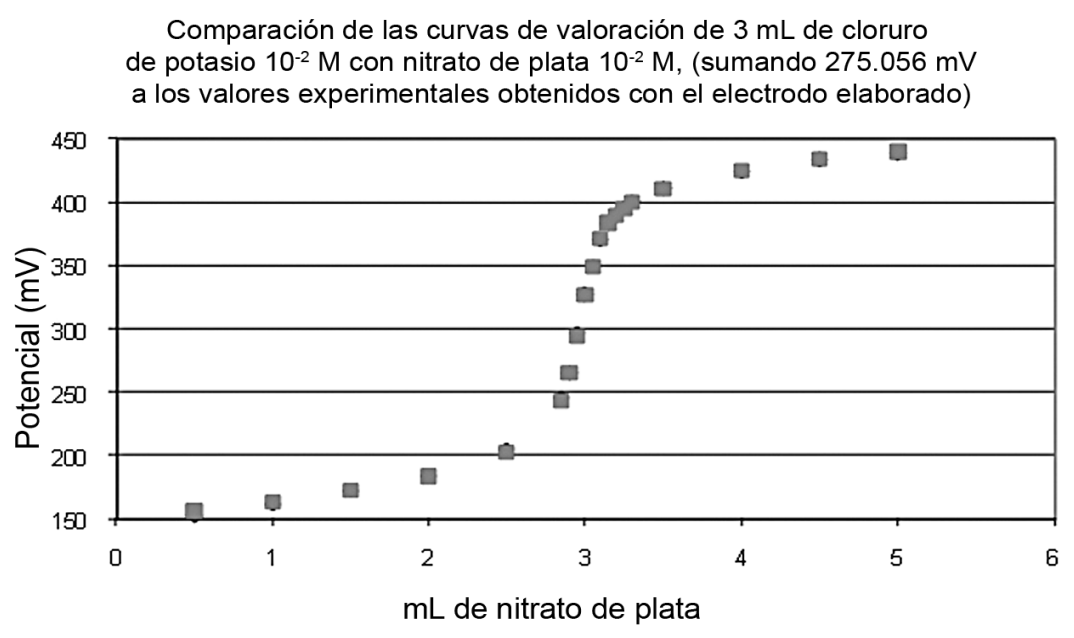

Figura 5. Comparación de las curvas de valoración promedio de cloruro de potasio 0.01 M con nitrato de plata 0.01 M, habiéndole sumado $275.056 \mathrm{mV}$ a los valores experimentales obtenidos con el electrodo elaborado.

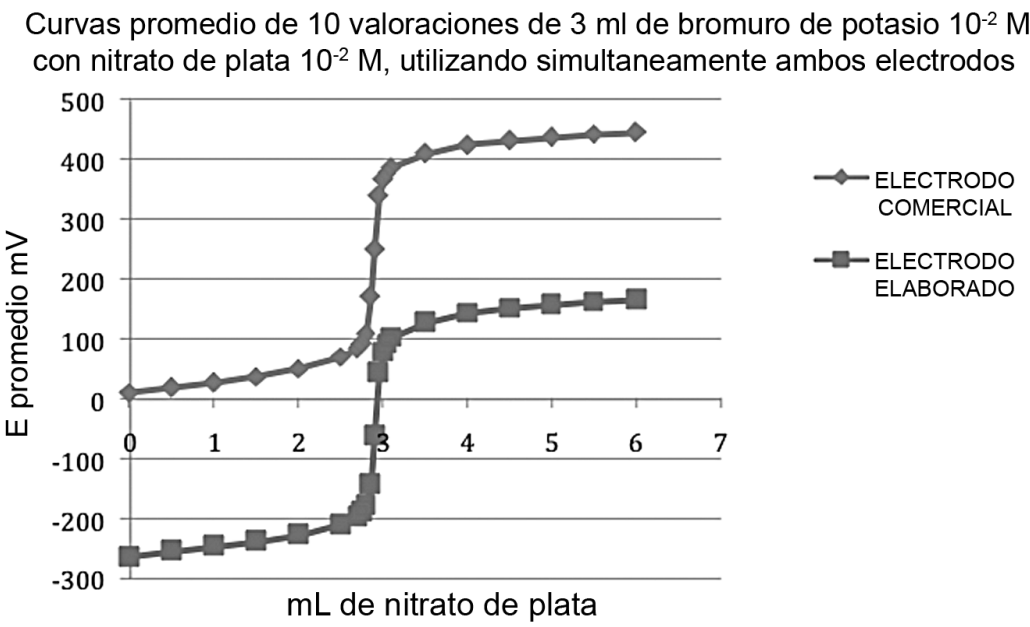

Figura 6. Curvas promedio de 10 valoraciones de bromuro de potasio $0.01 \mathrm{M}$ con nitrato de plata $0.01 \mathrm{M}$, utilizando simultáneamente electrodo comercial y electrodo elaborado.

\section{b. Electrodo de referencia cobre/nitrato de cobre:}

A un segundo tubo de las mismas características que el anterior se le introduce un alambre de cobre del mismo calibre y altura que el de plata, el cual es soldado al alambre de la cubierta del cable coaxial. Este segundo tubo se llena de nitrato de cobre, haciendo uso de una perforación lateral en su parte superior de 0,5 cm de diámetro, de igual manera que el electrodo de plata se sella con silicón. En la parte inferior se le coloca una fibra de algodón para permitir el contacto eléctrico. Los dos tubos se pegan uno al otro tal como se muestra en la figura I (Celdas electroquímicas, 2013).

Prueba del funcionamiento del electrodo elaborado mediante una curva de calibración

Una vez elaborado el electrodo, se hacen mediciones de potencial de equilibrio por triplicado con un potenciómetro Oakton modelo 356|4-80 de cuatro soluciones de nitrato de plata, reactivo analítico Merck de concentraciones $\left(10^{-1}, 10^{-2}, 10^{-}\right.$ ${ }^{3}, 10^{-4}$ ) molares (Orozco, 1997), colocando $10 \mathrm{~mL}$ 
Comparación de la curva de valoración de bromuro de potasio $0.01 \mathrm{M}$ con nitrato de plata $0.01 \mathrm{M}$ (sumando $284.74 \mathrm{mV}$ a los valores experimentales obtenidos con electrodo elaborado

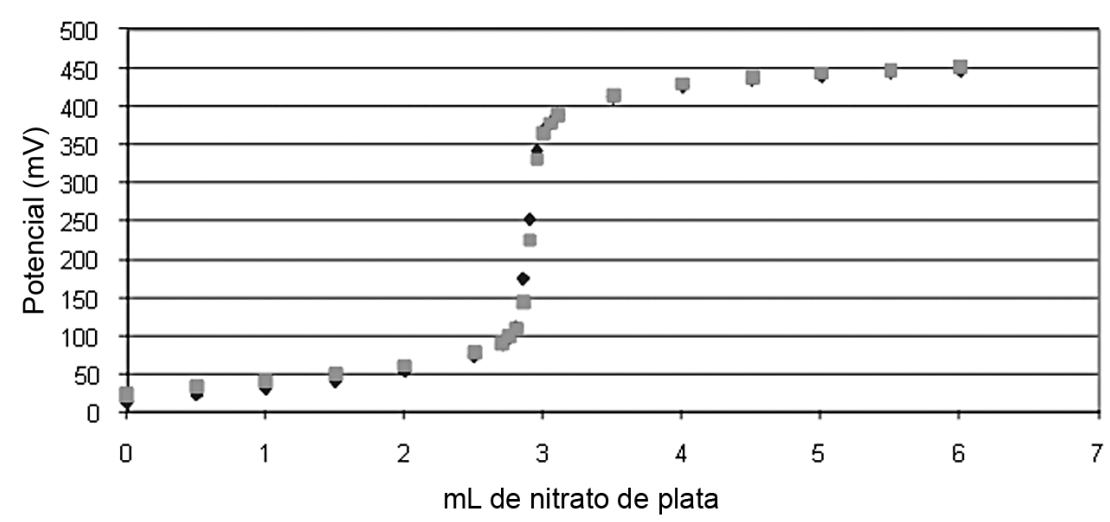

Figura 7. Comparación de las curvas de valoración promedio de cloruro de potasio 0.01 M con nitrato de Plata 0.01 M, habiéndole sumado $275.056 \mathrm{mV}$ a los valores experimentales obtenidos con el electrodo elaborado.

Cuadro I. Cálculo de la t de Student para comparar ambos métodos (Harris, 1991).

\begin{tabular}{|c|c|c|c|}
\hline Valoración & $S_{\text {combinada }}$ & $T_{\text {calculada }}$ & $T_{\text {tablas (95\%) }}$ \\
\hline De cloruro de potasio con nitrato de plata & 0.069 & 0.233 & 2.1 \\
\hline $\begin{array}{c}\text { De bromuro de potasio con nitrato } \\
\text { de plata }\end{array}$ & 0.094 & 1.04 & 2.1 \\
\hline
\end{tabular}

de las soluciones preparadas en vasos de $50 \mathrm{~mL}$, manteniendo una agitación constante (las reacciones se presentan en el Anexo I). Con los potenciales obtenidos se traza una curva de calibración (figura 2), utilizando la hoja de cálculo de Microsoft Office Excel 2007.

Comparación del funcionamiento del electrodo elaborado contra el electrodo convencional, en valoraciones de cloruro de potasio y bromuro de potasio.

\section{Valoración de cloruro de potasio}

De manera simultánea, con el electrodo combinado elaborado y el electrodo convencional se procedió a titular 10 veces una alícuota de $3 \mathrm{~mL}$ de cloruro de potasio $10^{-2} \mathrm{M}$ con nitrato de plata estandarizado $10^{-2} \mathrm{M}$. Como se muestra en la figura 3 (las reacciones se presentan en el Anexo 2), con los datos obtenidos se elabora la figura 4.
Valoración de bromuro de potasio

Procediendo experimentalmente de manera similar a la valoración de cloruro de potasio, se valora bromuro de potasio, obteniéndose la figura 6.

A los resultados obtenidos con el electrodo elaborado se le suman $284.74 \mathrm{mV}$, obteniéndose la figura 7 .

\section{Discusión y Conclusiones}

Se logró fabricar un electrodo combinado de plata como electrodo indicador y cobre/nitrato de cobre como referencia, que al evaluar su respuesta respecto a cuatro soluciones de nitrato de plata, reactivo analítico, de concentraciones $\left(10^{-1}, 10^{-2}, \mid 0^{-3}, 10^{-4}\right) \mathrm{M}$ se obtuvo una correlación $\left(r^{2}\right)$ de 0.999 y una pendiente $(\mathrm{m})$ tipo nernstiana de $68.89 \mathrm{mV}$, de respuesta rápida. Conociendo estos resultados, se cuantificó por 10 ocasiones $3 \mathrm{~mL}$ de cloruro de potasio $10^{-2}$ My por 10 ocasiones $3 \mathrm{~mL}$ de bromuro de potasio 
$10^{-2} \mathrm{M}$ con nitrato de plata $10^{-2} \mathrm{M}$, con un montaje paralelo, utilizando el electrodo elaborado y los electrodos convencionales (figura 3), encontrándose un comportamiento equivalente entre ambos sistemas (figuras 4 y 6 ).

Existe una diferencia de posición entre las gráficas, debido a que la referencia para el método convencional es un electrodo de plata/cloruro de plata y para el electrodo elaborado es de cobre/nitrato de cobre. Esta diferencia grafica se puede obviar, sumando los valores obtenidos con el electrodo elaborado por un factor de proporcionalidad de 275.056 para cloruro de potasio y de 284.74 para la valoración de bromuro de potasio (figuras 5 y 7 ).

De acuerdo con lo anteriormente mencionado y aplicando a los resultados experimentales de los volúmenes de punto de equivalencia obtenidos la prueba estadística de $t$ de Student con un nivel de confianza del 95\%, comprobamos que el electrodo elaborado funciona de manera equivalente a los electrodos utilizados en el montaje convencional ( $t$ experimental $0,233<\mathrm{t}$ de tablas $2, \mathrm{I}$, para la valoración de cloruro de potasio y $\mathrm{t}$ experimental 0,094 $<$ t de tablas 2, I, para la valoración de bromuro de potasio), puesto que no existe diferencia significativa.

De tal manera que el electrodo elaborado puede sustituir a los electrodos comerciales, evitándose el uso del puente de agar, además de que, por el diseño del electrodo, se pudieron realizar microvaloraciones ahorrándose reactivos, agua y disminuyendo la cantidad de desechos (Ibañez, 2000; Torres y Castellón, 2000). Habiéndose valorado cloruro de potasio y bromuro de potasio con el electrodo elaborado, se esperaría poder valorar yoduro de potasio, pero esto no es posible ya que la presencia de nitrato de cobre, debida al electrodo de referencia, provoca una anfolización de los iones cobre por la formación de yoduro de cobre (I), generando una interferencia en la formación del yoduro de plata (las reacciones se presentan en el Anexo 3).

\section{Agradecimientos}

Este trabajo se realizó con el apoyo del Programa UNAM-DGPA-PAPIME Clavel del proyecto PE2012/4.

A Draucin Jiménez Celi, por la elaboración de los tubos de vidrio con los que se elaboró el electrodo.

\section{Bibliografía}

Arnaiz, F. J. (2005). Química en microescala en los laboratorios de química inorgánica y organometálica. Educación química, 16(4), 504-508

Celdas electroquímicas. (2013). Clases. Recuperado de http: // www.ciens.ucv.ve/eqsol/Electroquimica/Clases/clases/P4.pdf

Charlot, G., Badoz-Lambling, J. \& Trémillon, B. (1969). Las reacciones electroquímicas. España:Toray-Masson.

Dean A., J. (Ed.). (1990). Manual de Química. McGraw Hill.

Harris, C. D. (1991). Análisis químico cuantitativo. México: Grupo Editorial Iberoamérica.

Ibáñez, J. G. (2000). La química en microescala en México: Hacia una panorámica general. Educación química, I | (I), |68-|7| .

Orozco, D. F. (1997). Análisis químico cuantitativo. México: Editorial Porrúa.

Pérez, S. J. de J., Rincón, A. S. \& Tenorio, M. H. (20/3). Construcción de un electrodo combinado posai-posai para microvaloraciones ácido-base. Tecnología en Marcha, 26(4).

Rincón, A. S. \& Pérez, S. J. de J. (2003). Potenciometría con volúmenes a nivel microescala en educación. Educación química, 14(3), |48-151.

Torres, E. E. \& Castellón, S. A. J. P. (2000). Minimización del impacto ecológico empleando microescala en los laboratorios de enseñanza química. Educación química, I I (2), 262-266.

\section{Anexo I}

Al utilizar como electrodo indicador un electrodo de plata metálica y ponerlo en una solución de nitrato de plata, se tiene el siguiente equilibrio electroquímico:

$$
\mathrm{Ag}^{+}+\mathrm{e}^{-} \longrightarrow \mathrm{Ag}^{\circ}
$$

Planteando la ecuación de Nernst correspondiente, queda:

$E=E^{\circ}+0,06 \log \left[\mathrm{Ag}^{+}\right]$

Ecuación de una línea recta, cuya pendiente $(m)$ es de 0,06 y ordenada al origen $E^{\circ}$, donde $E^{\circ}=0,8 v$

\section{Anexo 2}

I. La plata iónica $\left(\mathrm{Ag}^{+}\right)$, en presencia de los iones cloruro, forma un precipitado:

$$
\mathrm{Ag}^{+}+\mathrm{Cl}^{-} \longleftrightarrow \mathrm{AgCl}_{\text {sólido }}
$$


Con una constante de producto de solubilidad Ks de: $\left[\mathrm{Ag}^{+}\right]\left[\mathrm{Cl}^{-}\right]=10^{-10}$

Si de la ecuación del Ks despejamos $\left[\mathrm{Ag}^{+}\right]$y la sustituimos en la ecuación (I) queda:

$\mathrm{E}=0,8+0,06 \log \left[\mathrm{IO}^{-10}\right] /\left[\mathrm{Cl}^{-}\right]=0,2+\log \mathrm{I} /[\mathrm{Cl}]$

O bien:

$\mathrm{E}=0,2+\mathrm{pCl}$

Donde el potencial medido es función lineal de $\mathrm{pCl}$

2. La plata iónica $\left(\mathrm{Ag}^{+}\right)$, en presencia de los iones bromuro, forma un precipitado:

$$
\mathrm{Ag}^{+}+\mathrm{Br}^{-} \rightleftarrows \mathrm{AgBr}_{\text {sólido }}
$$

Con una constante de producto de solubilidad Ks de:

$\left[\mathrm{Ag}^{+}\right][\mathrm{Br}]=10^{-12}$

Como en el caso de los cloruros, para los bromuros:

$\mathrm{E}=0,08+\mathrm{pBr}$

Donde el potencial medido es función lineal de pBr

\section{Anexo 3}

Los potenciales normales de los sistemas del cobre (Langes 1990) son:

$$
\begin{array}{r}
\mathrm{Cu}^{2+}+\mathrm{e}^{-} \longrightarrow \mathrm{Cu}^{+} \\
\mathrm{E}^{\circ}=0,17+0,06 \log \left[\mathrm{Cu}^{2+}\right] /\left[\mathrm{Cu}^{+}\right] . \\
\mathrm{Cu}^{2+}+\mathrm{e}^{-} \longrightarrow \mathrm{Cu}^{\circ}
\end{array}
$$$$
E^{\circ}=0,52+0,06 \log \left[\mathrm{Cu}^{+}\right]
$$

Colocados en una escala de predicción de reacción:

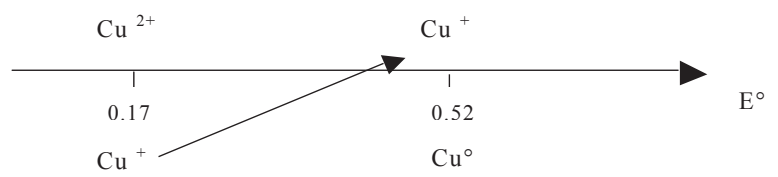

Se puede observar que el ión cobre (I) dismuta.

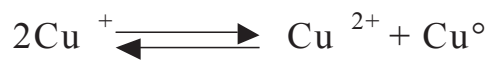

Con una constante de dismutación (Kd) de $10^{5.83}$
En presencia de yoduros, el ión cobre (I) reacciona formando un precipitado.

$$
\mathrm{Cu}^{+}+\mathrm{I}^{-} \rightleftarrows \mathrm{CuI} \text { sólido }
$$

Con una constante de producto de solubilidad Ks de:

$\left[\mathrm{Cu}^{+}\right]\left[\mathrm{I}^{-}\right]=10^{-12}$

Con este valor de Ks y las ecuaciones (2) y (3) se pueden calcular los valores de potencial de los pares electroquímicos $\mathrm{Cul}_{\text {sólido }} / \mathrm{Cu}^{\circ}$ y $\mathrm{Cu}^{2+} / \mathrm{Cul}{ }_{\text {sólido' }}$ de la siguiente manera, para el par $\mathrm{Cu}^{2+} / \mathrm{Cul}_{\text {sólido: }}$

Despejando $\left[\mathrm{Cu}^{+}\right]$de la ecuación (4) queda:

$\left[\mathrm{Cu}^{+}\right]=10^{-12} /\left[\mathrm{I}^{-}\right]$

Sustituyendo (5) en la ecuación (2) y operando algebraicamente queda:

$E=0,17+0,06 \log 10^{12}+0,06 \log \left[\mathrm{Cu}^{2+}\right]\left[\mathrm{I}^{-}\right]$

Operando algebraicamente:

$E=0,89+0,06 \log \left[\mathrm{Cu}^{2+}\right]\left[\mathrm{I}^{-}\right]$.

Para el par Cul sólido $/ \mathrm{Cu}^{\circ}$, sustituyendo (5) en la ecuación (3) y operando algebraicamente queda:

$E=0,52+0,06 \log 10^{-12}+0,06 \log 1 /\left[\mathrm{I}^{-}\right]$

Obien:

$E=-0,20+0,06 \log \mid /\left[\mathrm{l}^{-}\right]$

Colocando los valores obtenidos en las ecuaciones (6) y (7) en una nueva escala de predicción de reacción se tiene:

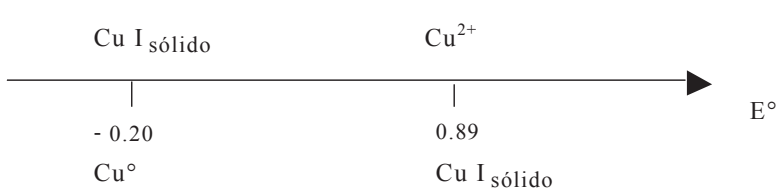

Donde se observa que el ión cobre (II) se anfoliza como se muestra en la nueva escala de predicción, estabilizándose el ion cobre (I) como Cul sólido Con:

$$
\mathrm{Cu}^{\circ}+\mathrm{Cu}^{2+}+2 \mathrm{I}^{-} \longleftrightarrow 2 \mathrm{CuI} \text { sólido }
$$

$Y$ una Keq $=10^{18,16}$

Generándose un equilibrio interferente para la valoración de ión yoduro. 